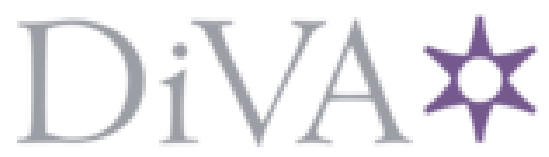

http://www.diva-portal.org

This is the published version of a paper presented at Engineering in Medicine and Biology Society (EMBC), 2015 37th Annual International Conference of the IEEE.

Citation for the original published paper:

Abtahi, F., Ji, G., Lu, K., Rodby, K., Seoane, F. (2015)

A knitted garment using intarsia technique for Heart Rate Variability biofeedback: Evaluation of initial prototype.

In: Engineering in Medicine and Biology Society (EMBC), 2015 37th Annual International Conference of the IEEE (pp. 3121-3124).

http://dx.doi.org/10.1109/EMBC.2015.7319053

N.B. When citing this work, cite the original published paper.

Permanent link to this version:

http://urn.kb.se/resolve?urn=urn:nbn:se:kth:diva- 179906 


\title{
A Knitted Garment using Intarsia Technique for Heart Rate Variability Biofeedback: Evaluation of Initial Prototype
}

\author{
F. Abtahi, Student Member, IEEE, G. Ji, K. Lu, K. Rödby, and F. Seoane, Senior Member, IEEE
}

\begin{abstract}
Heart rate variability (HRV) biofeedback is a method based on paced breathing at specific rate called resonance frequency by giving online feedbacks from user respiration and its effect on HRV. Since the HRV is also influence by different factors like stress and emotions, stress related to an unfamiliar measurement device, cables and skin electrodes may cover the underling effect of such kind of intervention. Wearable systems are usually considered as intuitive solutions which are more familiar to the end-user and can help to improve usability and hence reducing the stress. In this work, a prototype of a knitted garment using intarsia technique is developed and evaluated. Results show the satisfactory level of quality for Electrocardiogram and thoracic electrical bioimpedance i.e. for respiration monitoring as a part of HRV biofeedback system. Using intarsia technique and conductive yarn for making the connection instead of cables will reduce the complexity of fabrication in textile production and hence reduce the final costs in a final commercial product. Further development of garment and Android application is ongoing and usability and efficiency of final prototype will be evaluated in detail.
\end{abstract}

\section{INTRODUCTION}

Wearable solutions for pervasive measurements of biological signals are considered as a potential solution for conditions arisen from the aging population. The increasing elder population together with better diseases prognosis increase the number of patients suffering from a chronic condition who require healthcare resources. Only implementing preventive healthcare, supporting healthy citizens to keep an active and healthy aging and providing personalized disease management and out-hospital care to chronic patient will impede that the healthcare cost will become unsustainable. Prevention and change in lifestyle could be a key solution for decreasing the costs by reducing the demand for treatment. Thus, both elderly patients with chronic conditions and healthy aging citizens can benefit from pervasive and home monitoring solutions for

*Research supported by Swedish Knowledge foundation project 20110136, and by Vinnova (The Swedish Governmental. Agency for Innovation Systems) through the Vinn Excellence program for IPack center and the Smart Textiles research and innovation environment.

F. Abtahi is with the School of Technology and Health, KTH Royal Institute of Technology, 14152 Huddinge, Sweden and the Department of Clinical Science, Intervention and Technology, Karolinska Institute, 14186 Stockholm, Sweden (e-mail: farhad.abtahi@sth.kth.se).

F. Seoane is with the Faculty of Caring Science, Working life and Social Welfare, University of Borås, SE-50190 Borås, Sweden and School of Technology and Health, KTH Royal Institute of Technology, 14152 Huddinge, Sweden. (e-mail: fernando.seoane@hb.se).

G. Ji and K. Lu are with the School of Technology and Health, KTH Royal Institute of Technology, 10044 Stockholm, Sweden.

$\mathrm{K}$. Rödby is with the Faculty of Textiles, Engineering and Business at University of Borås, SE-50190 Borås, Sweden management of chronic diseases and keeping a healthy lifestyle. There is an intuitive agreement in the research community that wearable systems can improve the usability in homecare and wellness by replacing the sticky electrodes and cables with textile electrodes and connections embedded in the clothing.

As a part of a project to evaluate Heart Rate Variability (HRV) biofeedback intervention by using a developed Android application [1], we have observed the stress of putting traditional ECG electrodes and associated cables as a threat which may cover the underling improvement in balance of autonomic nervous system i.e. higher HRV and lower heart rate. Therefore, we are developing a wearable solution with textile electrodes and connections to the recorder embedded in a garment. Most common method of connecting textrodes, textile electrodes, to a recorder unit is by using conventional cables e.g. the sensorized garments in the ATREC project [2] and the textrode straps for impedance cardiography [3] or even commercial solutions like Hexoskin $T$-shirt, Equivital vest. In this work, knitted conductive yarn using intarsia technique is evaluated for the future use in garments for measurement of Electrocardiogram (ECG) and electrical bioimpedance (EBI) e.g. for monitoring the respiration through thoracic $E B I$.

\section{METHOD}

\section{A. HRV Biofeedback}

HRV refers to variation of heart rate between consecutive heart beats. Since heart rate is controlled by autonomic nervous system (ANS), heart rate fluctuations can be used to monitor ANS. ANS consists of two branches called parasympathetic, rest and digest, and sympathetic, fight and flight. HRV is the result of several feedbacks to the heart through sympathetic/parasympathetic branches e.g. stresses blood pressure, respiration, emotions and thermoregulation. $\mathrm{HRV}$ reduce by age and reduced HRV is also associated with chronic diseases and bad outcome e.g. diabetes, systematic inflammation [4], depression [5] and sudden cardiac death [6]. Therefore, it seems beneficial to investigate intervention methods to increase the HRV for obtaining ANS balance.

HRV biofeedback is a potential method for increasing HRV and improving balance of ANS. The idea is to breath at a specific individual rate called resonance frequency. Resonance frequency is the rate of feedback to the heart in order to elevate/lower the heart rate because of drop/rise in the blood pressure, respectively. By breathing at this rate, feedback from respiration and blood pressure will have coherence and hence more efficient. This may enhance the baroreflex gain and improve the $\operatorname{HRV}[7,8]$. 

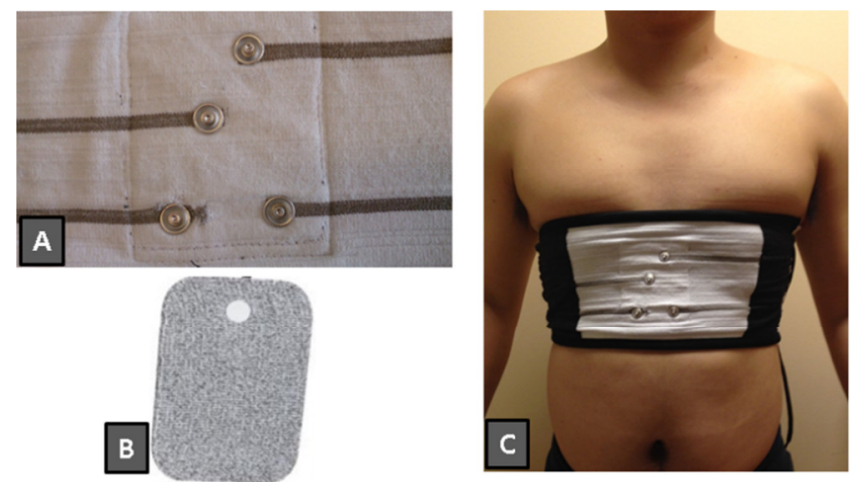

Figure 1. Prototype of supporting garment with conductive yarn (gray traces) knitted with intarsia technique (A), Dacron textrode (B) and garment worn by a subject (C).

\section{B. Textrodes}

An elastic knitted Electro-Mesh ${ }^{\mathrm{TM}}$ electrode with silver treated nylon fibers blended with Dacron ${ }^{\mathrm{TM}}$ (Prizm Medical, Inc., Duluth, Georgia) with size of $4 \times 5 \mathrm{~cm}$ is chosen. The high conductivity of whole fabric glued in this textrodes make it easier to collect high quality signals even without applying a conductive spray nor wetting them, as it is customary to do when using textrodes for signal recording.

\section{Knitting using Intarsia Technique}

Knitting of conductive yarn is a common technique for making conductive fabrics used in Eletro-Mesh or Shieldex ${ }^{\circledR}$ Fabric P130+B (STATEX Gmbh., Bremen, Germany). In this work a garment manufactured using the intarsia technique [9] has been knitted at School of Textiles at the University of Borås. Figure 1 shows the knitted prototype. The gray traces are the conductive segments connecting the electrodes to the measurement device. Garment is providing pressing studs for placing and inter-connecting the four electrode/ textrode needed for one-lead ECG and tetra-polar thoracic EBI measurement i.e. using a pair of electrodes for
TABLE I. SUMMARY OF MEASUREMENTS

\begin{tabular}{|c|c|c|c|c|l|}
\hline Title & $\mathbf{3 M}$ & Textrode & Cable & Intarsia & \multicolumn{1}{c|}{ Comparison } \\
\hline Case 1 & $\checkmark$ & $\times$ & $\checkmark$ & $\times$ & $\begin{array}{l}\text { Offline } \\
\text { Matlab }\end{array}$ \\
\hline Case 2 & $\checkmark$ & $\times$ & $\times$ & $\checkmark$ & $\begin{array}{l}\text { Offline } \\
\text { Matlab }\end{array}$ \\
\hline Case 3 & $\times$ & $\checkmark$ & $\checkmark$ & $\times$ & $\begin{array}{l}\text { Offline } \\
\text { Matlab }\end{array}$ \\
\hline Case 4 & $\times$ & $\checkmark$ & $\times$ & $\checkmark$ & $\begin{array}{l}\text { Offline } \\
\text { Matlab }\end{array}$ \\
\hline Case 5 & $\times$ & $\checkmark$ & $\times$ & $\checkmark$ & $\begin{array}{l}\text { Online } \\
\text { HRV Biofeedback }\end{array}$ \\
\hline
\end{tabular}

Note: Measurements are done in the first four scenarios: normal breathing, paced breathing and holding the breath.

injecting the current and another pair for sensing the voltage.

\section{Experimental Setup}

Performance of garment and textrode for HRV biofeedback are evaluated in three steps: (1) performance of intarsia connection compare to conventional cables, (2) performance of textrodes compare to diagnostic ECG 3M red-dot ${ }^{\mathrm{TM}} 2670-5 \mathrm{Ag} / \mathrm{AgCl}$ electrodes $(4 \mathrm{~cm} \times 3 \mathrm{~cm}$ ), and (3) performance of complete textile, textrode and intarsia connection, compare to conventional solution, $3 \mathrm{M}$ and standard cables. Table 1 is summarizing the combination of electrode/textrodes and intarsia/cable connections.

The measurements are done by using a Bluetooth device and windows application. The device, is designed by Z-Health Technologies $\mathrm{AB}$ and has been used in previous studies [1, 2], it measures both Electrocardiogram (ECG) and respiration activity. For this study the sampling frequency is set to 300 $\mathrm{Hz}$ for both ECG/EBI and thoracic EBI is done with the injecting current at $50 \mathrm{kHz}$. Electrode placements on the chest were marked to minimize the effect of electrode location during the experiment. The subjects are asked to breathe normally for two minutes, controlling the breathing by using a metronome for one minute and finally keeping their breath for 15 seconds. In the next step, usability of garment for HRV biofeedback is evaluated by asking subjects

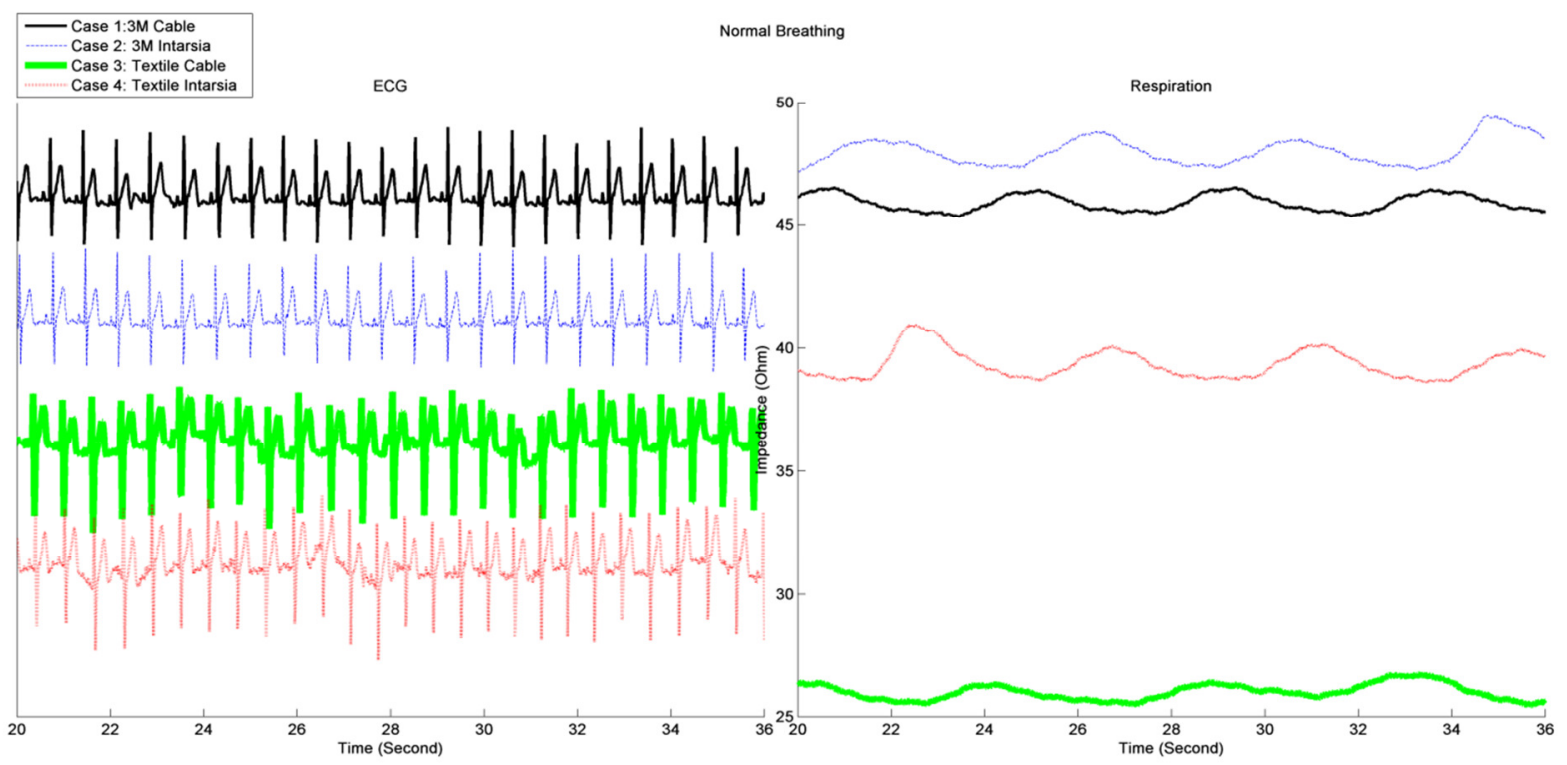

Figure 2. 10-seconds epoch of measured ECG (left) and thoracic bioimpedance (right) during normal breathing. Signals collected in Case 1-4, are depicted by black solid line, blue dashed, green thick line and red dotted, respectively. 


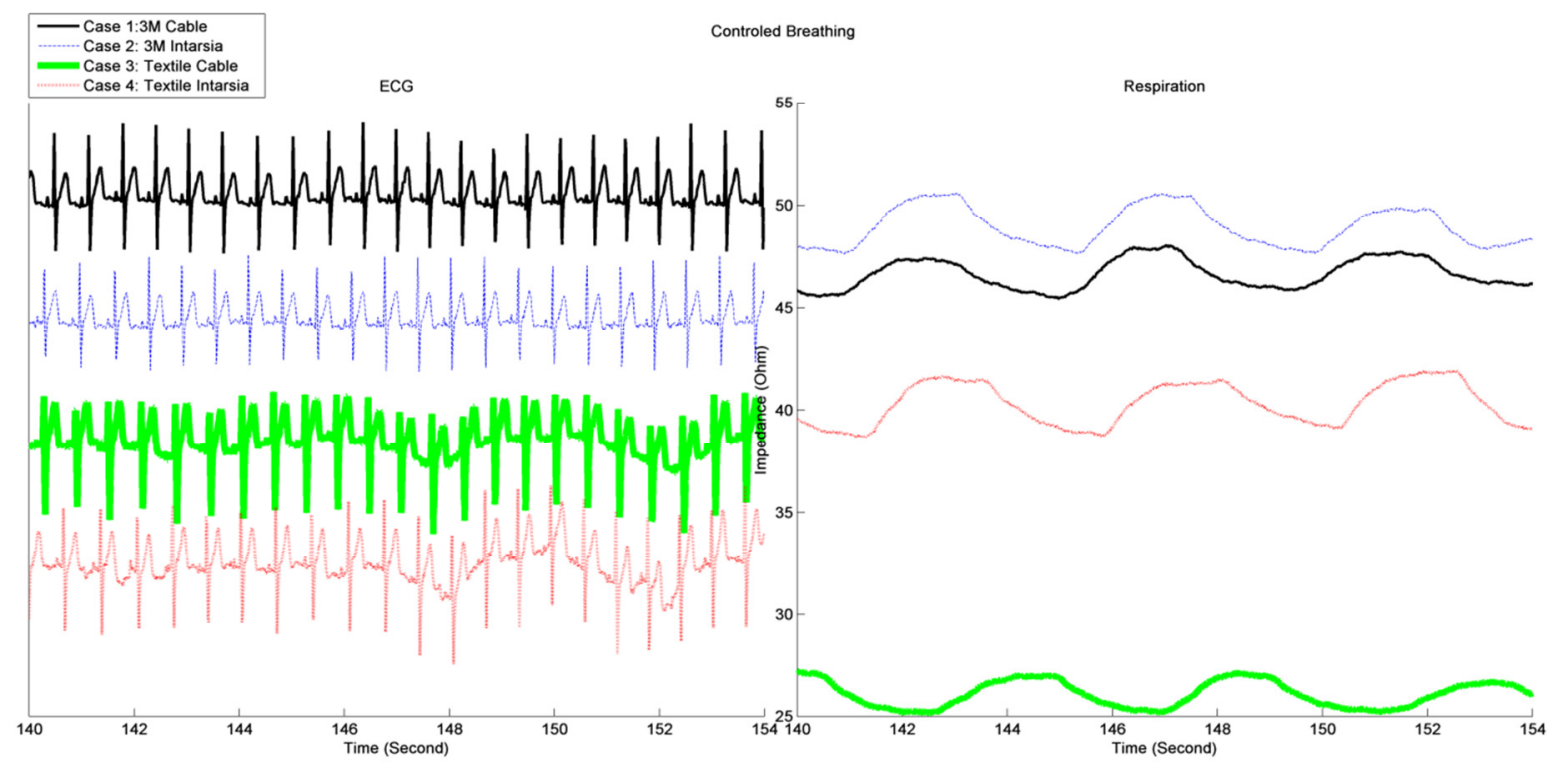

Figure 3. 10-seconds epoch of measured ECG (left) and thoracic bioimpedance (right) during controlled breathing by using metronome. Signals collected in Case 1-4, are depicted by black solid line, blue dashed, green thick line and red dotted, respectively.

to use the developed Android application.

All the recordings are saved and imported into Matlab for further processing. All collected signals are similarly preprocessed for removing the powerline noise and baseline wander.

\section{RESULTS}

\section{A. Comparison of measurements}

Measurements by using prototype garment are done in four cases, see Table 1. The ECG and thoracic bioimpedance signals measured during normal, controlled and hold berthing is illustrated in Figure 2-4, respectively. It can be clearly seen from the plots that ECG signals obtained with combination of textrode and intarsia, produce an ECG reassembling a V3-V4 lead with enough good quality for the R-peak detection
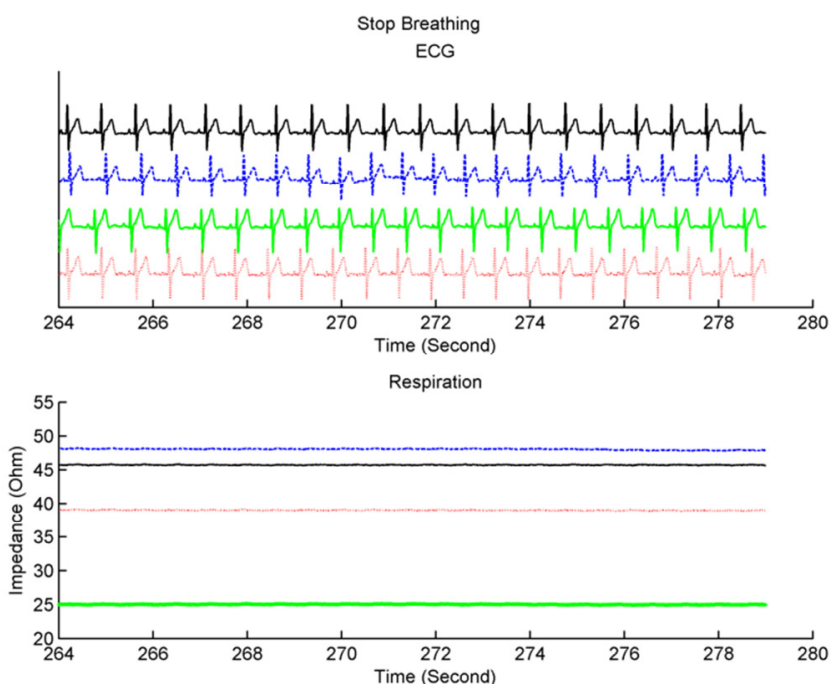

Figure 4. 15-seconds epoch of measured ECG and thoracic bioimpedance during holed breath; Signals collected in Case 1-4, are depicted by black sold, blue dashed, green dash-dot and red dotted, respectively. required in HRV biofeedback applications.

Regarding the thoracic EBI recordings It can be seen that highest baseline is obtained for the cases where the textrode is used while and the lowest baseline is obtained for the $3 \mathrm{M}$ electrodes Despite the different baseline value for the thoracic bioimpedance, the TBI signals in all four cases clearly show the rhythmic deviation of respiration activity.

\section{B. Usability for HRV Biofeedback}

Subjects successfully managed to use the system by using the prototype garment shown in Figure 1.C. In Figure 5 it is shown a screenshot of the Android biofeedback application showing the target breathing activity and the current respiration activity assessed by the Thoracic bioimpedance measurement. The ECG, the Heart rate and the HRV as expressed by the power spectral density is also displayed.

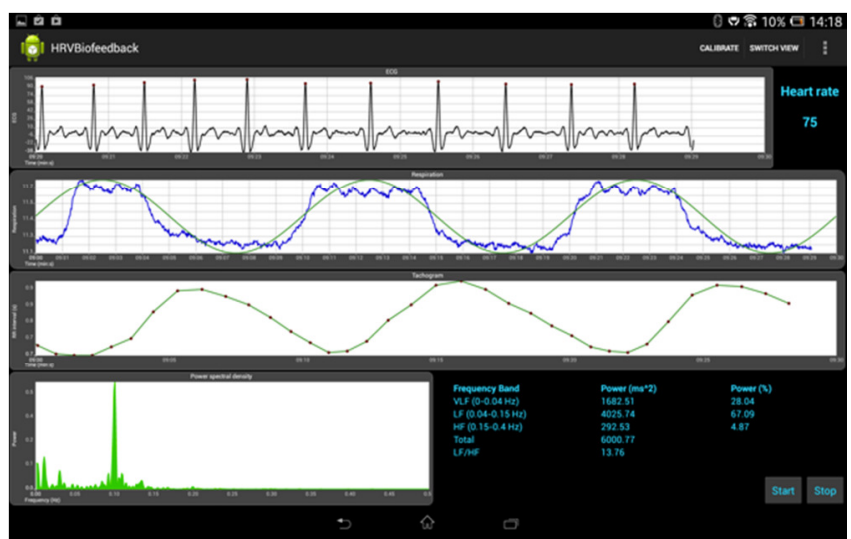

Figure 5. Default view of biofeedback application, showing from top to bottom: ECG, respiration, tachogram and power spectral density. The bottom right corner shows the power in different frequency bands. The maximum peak in the power spectrum at $0.1 \mathrm{~Hz}$ corresponds to a respiration. 


\section{DISCUSSION AND CONCLUSION}

It is possible to evaluate from both the ECG and the thoracic EBI recordings plotted in Figures 2-4, the quality of biosignals recorded with the intarsia garment and textile electrodes is comparable to recordings obtained with conventional gel electrodes and wire connections.

The shape of the QRS, similar to a V3-V4 lead, obtained with the garment is consistence with the placement of the electrodes. It is possible to see a very slight difference in the height of the $\mathrm{R}$ and $\mathrm{S}$ complex that is smaller for cases $3 \& 4$, similar to a V3 lead than for cases $1 \& 2$ similar to V4. This is due to the fact that the electrodes for case $1 \& 2$, gel, different than for case $3 \& 4$, textrodes. Although meant to be in the exact same position, the fact is that the size of the textrodos is $20 \mathrm{~cm}^{2}$, significantly larger the $10 \mathrm{~cm}^{2}$ area of the $3 \mathrm{M}$ gel electrodes. In addition to the size issue, it is also a fact that the gel electrodes have the press stud in the precise centre but the used textrodes has the press stud placed in the centre but slightly shifted to one side, see Figure 1.B

The differences in the baseline value of thoracic EBI measurements also differ for the different case 1-4. When using gel electrodes the baseline values are very similar as shown in Figure 4. It is when the textrodes are used the differences are remarkable. The main reasons for these differences are precisely the reason introduced in the previous paragraph, difference size and slightly different placement on the chest due to the press stud small difference.

Another influence worth mentioning related to different between electrodes caused by the different nature of the materials, gel versus dry; it is the influence of breathing on the baseline of the ECG recordings. This influence is seen straightaway in Figures 3 and 4 presenting plots for cases $1 \&$ 2 with an ECG baseline completely stable but a noticeable ripple in the baseline of the ECG recorder for cases $3 \& 4$. The main responsible for the ripple is the changes in the skinelectrode interface caused by the friction between the skin and the textrode fabric cause by breathing. Notice that when breath is hold the ripple disappears, as shown in Figure 5, where all 4 ECG recordings are free from any ripple.

Regarding the using intarsia knitted connections and textrodes, we must say the observed influence on the biosignals recordings related to the use of intarsia connections is negligible in comparisons of the influence of using textrodes, even textrodes that have successfully worked without requiring wetting the interface; however, the quality of the recording will depend on natural dryness of subject's skin and own sweat production.

The evaluation of prototype garment has been done for the specific use in a specific HRV biofeedback application. Since a biofeedback session is done in sitting position and during the rest. Motion artefacts are not playing the main role as the usually do in long term ECG monitoring e.g. sport performance and exercise monitoring or holter recording for atrial fibrillation detection. Therefore, the study of garment performance during major movements e.g. walking and running has not been done for considering it not useful for the application.
The use of intarsia technique for making the conductive fabric and connections were originally introduced in the Wealthy project one decade ago [10], Since then other authors like L. Li et al. [11] have reported successful uses of intarsia for knitting sensorized garments. However, the use of cables embedded in textiles still the method of choice to establishing electrical connections in the commercially available products e.g Hexoskin T-shirt, Equivital vest. Such textile un-friendly method makes the manufacturing process of such a garments more difficult and time consuming and thereupon more expensive. We believe that integrating the electrical connections on the same fabrics used to manufacture the garments can help to improve the manufacturing of wearable biomedical sensors and system towards the affordability expected in clothing and textile apparel.

\section{REFERENCES}

F. Abtahi, A. Berndtsson, S. Abtahi, F. Seoane, and K Lindecrantz, "Development and preliminary evaluation of an Android based heart rate variability biofeedback system," in Engineering in Medicine and Biology Society (EMBC), 2014 36th Annual International Conference of the IEEE, 2014, pp. 3382-3385.

[2] F. Seoane, J. Ferreira, L. Alvarez, R. Buendia, D. Ayllón, C. Llerena, et al., "Sensorized Garments and Textrode-Enabled Measurement Instrumentation for Ambulatory Assessment of the Autonomic Nervous System Response in the ATREC Project," Sensors, vol. 13, pp. 8997-9015, 2013.

[3] J. C. M. Ruiz, M. Rempfler, F. Seoane, and K. Lindecrantz, "Textrode-enabled transthoracic electrical bioimpedance measurements-towards wearable applications of impedance cardiography," Journal of Electrical Bioimpedance, vol. 4, pp. 45-50, 2013

[4] K. J. Ellis, S. J. Bell, G. M. Chertow, W. C. Chumlea, T. A. Knox, D. P. Kotler, et al., "Bioelectrical impedance methods in clinical research: a follow-up to the NIH Technology Assessment Conference," Nutrition, vol. 15, pp. 874-880, 1999.

[5] R. M. Carney, K. E. Freedland, and R. C. Veith, "Depression, the autonomic nervous system, and coronary heart disease," Psychosomatic medicine, vol. 67, pp. S29-S33, 2005.

[6] M. T. La Rovere, G. D. Pinna, R. Maestri, A. Mortara, S Capomolla, O. Febo, et al., "Short-term heart rate variability strongly predicts sudden cardiac death in chronic heart failure patients," circulation, vol. 107, pp. 565-570, 2003.

[7] P. M. Lehrer, E. Vaschillo, and B. Vaschillo, "Resonant frequency biofeedback training to increase cardiac variability: Rationale and manual for training," Applied psychophysiology and biofeedback, vol. 25, pp. 177-191, 2000.

[8] P. M. Lehrer, "Biofeedback training to increase heart rate variability," Principles and practice of stress management, vol. 3, pp. 227-248, 2007.

[9] W. Zhi, "The Structure and Forming Principle of Knitted Intarsia Fabrics," Knitting Industries, vol. 6, p. 013, 1987.

[10] R. Paradiso, G. Loriga, and N. Taccini, "A wearable health care system based on knitted integrated sensors," Information Technology in Biomedicine, IEEE Transactions on, vol. 9, pp. 337-344, 2005.

[11] L. Li, W. M. Au, Y. Li, K. M. Wan, S. H. Wan, and K. S. Wong, "Design of intelligent garment with transcutaneous electrical nerve stimulation function based on the intarsia knitting technique," Textile Research Journal, 2009. 